\title{
T1 Gadolinium Enhancement of Intracranial Atherosclerotic Plaques Associated with Symptomatic Ischemic Presentations
}

\author{
P. Vakil, J. Vranic, M.C. Hurley, R.A. Bernstein, A.W. Korutz, A. Habib, A. Shaibani, F.H. Dehkordi, T.J. Carroll, and S.A. Ansari
}

\begin{abstract}
BACKGROUND AND PURPOSE: Contrast enhancement of intracranial atherosclerotic plaques has recently been investigated using high field and high resolution MR imaging as a risk factor in the development of ischemic stroke. We studied the reliability of conventional MR imaging at 1.5T in evaluating intraplaque enhancement and its relationship with acute cerebrovascular ischemic presentations in patients with severe intracranial atherosclerotic disease.
\end{abstract}

MATERIALS AND METHODS: We retrospectively identified and analyzed 19 patients with 22 high-grade intracranial atherosclerotic disease plaques ( $>70 \%$ stenosis) in vessels cross-sectionally visualized by neuroanatomic MR imaging. Atherosclerotic plaques were classified as asymptomatic or symptomatic. Two blinded neuroradiologists independently ranked each lesion for the presence of intraplaque enhancement by use of a 5-point scale (1-5). Furthermore, plaque enhancement was quantified as the relative change in T7WI spin-echo signal intensity (postcontrast/precontrast) in the vessel wall at the site of each intracranial atherosclerotic disease lesion.

RESULTS: Intraplaque enhancement was observed in 7 of 10 (70\%) symptomatic plaques, in contrast to 1 of 12 (8\%) asymptomatic plaques. Interobserver reliability correlated well for intraplaque enhancement $(\kappa=0.82)$. The degree of relative plaque enhancement in symptomatic versus asymptomatic lesions (63\% versus $23 \%$ ) was statistically significant $(P=.001, t$ test).

CONCLUSIONS: In this pilot study, we determined that intraplaque enhancement could be reliably evaluated with the use of crosssectional imaging and analysis of vessels/plaques by use of conventional neuroanatomic MR imaging protocols. In addition, we observed a strong association between intraplaque enhancement in severe intracranial atherosclerotic disease lesions and ischemic events with the use of conventional MR imaging. Our preliminary study suggests that $\mathrm{Tl}$ gadolinium-enhancing plaques may be an indicator of progressing or symptomatic intracranial atherosclerotic disease.

ABBREVIATIONS: ICAD = intracranial atherosclerotic disease; IPE = intraplaque enhancement; Tlw-SE = TI-weighted spin-echo; IPH = intraplaque hemorrhage; $\mathrm{ACA}=$ anterior cerebral artery

ntracranial atherosclerotic disease (ICAD) is a significant cause of stroke worldwide, accounting for $7-10 \%$ of acute ischemic stroke cases. ${ }^{1}$ In symptomatic ICAD with high-grade stenoses

Received October 1, 2012; accepted after revision March 7, 2013.

From the Departments of Radiology (P.V., J.V., M.C.H., A.W.K., A.H., A.S., T.J.C., S.A.A.), Neurology (R.A.B., S.A.A.), Neurosurgery (M.C.H., A.S., S.A.A.), and Biomedical Engineering (P.V., T.J.C.), Northwestern University, Feinberg School of Medicine, Chicago, Illinois; and Department of Economics and Decision Sciences (F.H.D.), Western Illinois University, Macomb, Illinois.

Please address correspondence to Sameer A. Ansari, MD, PhD, Department of Radiology, Neurology, and Neurosurgery, Northwestern University, Feinberg School of Medicine, 676 N St Clair St, Suite 800, Chicago, IL 60611-2927; e-mail: s-ansari@northwestern.edu

This study was supported by NIH/NHLBI grants R01 HL088437 and NIH/NIBIB T32 EB005170.

O- Indicates open access to non-subscribers at www.ajnr.org

三 Indicates article with supplemental on-line table.

http://dx.doi.org/10.3174/ajnr.A3606
$(>70 \%)$, the risk of stroke or death at 1 year is $12-22 \%$ with medical therapy based on the WASID ${ }^{1}$ (Warfarin-Aspirin Symptomatic Intracranial Disease) and SAMMPRIS ${ }^{2}$ (Stent placement versus Aggressive Medical Management for Preventing Recurrent Stroke in Intracranial Stenosis) trial.

The SAMMPRIS trial was designed to determine whether intracranial angioplasty/stent placement as a primary treatment option could benefit patients with severe ICAD in reducing the risk of stroke/death over aggressive medical management alone. However, patient recruitment in the SAMMPRIS trial was prematurely terminated as mandated by the National Institutes of Health/National Institute of Neurological Disorders and Stroke because of unexpectedly high stroke/death rates with endovascular intervention $(14.7 \%$ at 30 days and $20 \%$ at 1 year) and better-than-expected outcomes with modern medical management $(5.8 \%$ at 30 days and $12.2 \%$ at 1 year). ${ }^{2}$ Hence, the role of intracranial angioplasty/stent placement was again relegated to a subpopulation of 
patients with severe ICAD who would fail optimum medical therapy. Although these patients remain at risk for recurrent TIAs or strokes, we have yet to develop a stratification scheme to identify this refractory subpopulation and perhaps alter their management (medical and/or surgical) before an ischemic presentation.

Increasing evidence indicates the role of neovascularization and intraplaque hemorrhage (IPH) in the progression and rupture of carotid atherosclerotic plaques analogous to the coronary literature. ${ }^{3,4}$ Inflammation, which is associated with neovascularization and IPH, locally alters vascular endothelium permeability, potentially allowing gadolinium contrast uptake and visualization on T1WI. In fact, evidence of neovascularization and/or focal inflammation in the arterial wall of the cervical carotid arteries through atherosclerotic plaque enhancement has been correlated with a higher incidence of ipsilateral stroke secondary to extracranial atherosclerotic lesions. ${ }^{3,5,6}$ Spagnoli et $\mathrm{al}^{7}$ recently reported on the high-resolution MR imaging findings of both IPH and adventitial enhancement (neovascularization and/or inflammation) as being independently associated with symptomatic carotid stenoses and recent cerebrovascular ischemic events.

Inflammation may be an attractive imaging marker in the setting of ICAD for risk stratification, though the diminutive nature of intracranial vessels makes plaque evaluation challenging because of spatial resolution and signal-to-noise ratio concerns. Several groups have attempted high-field, high-spatial resolution, contrast-enhanced MR imaging of the intracranial vessel wall and atherosclerotic plaques with the use of dark-blood sequences. ${ }^{8-13}$ Swartz et $\mathrm{al}^{9}$ identified the existence of intracranial atherosclerotic plaque enhancement through the use of $3 \mathrm{~T}$ high-resolution MR imaging. More recently, this type of high-resolution MR imaging assessment found strong enhancement in ICAD plaques in patients with acute ischemic stroke. ${ }^{14}$ In this pilot study, it was our goal to determine whether postgadolinium T1 intracranial plaque enhancement (IPE) could be reliably delineated through the use of low-resolution, conventional neuroanatomic MR techniques in patients with severe ICAD (>70\% stenosis) and whether IPE was associated with the occurrence of recent ischemic cerebrovascular events.

\section{MATERIALS AND METHODS Patients}

Institutional review board approval was obtained for a retrospective study to identify patients with severe ICAD causing $\geq 70 \%$ stenosis, diagnosed with our institution's MR imaging/MRA stroke protocol between January 2008 and January 2011. A PACS-based report search was performed on this cohort to identify subjects with severe ICAD. The following key word search terms were used: "severe OR high-grade OR attenuation" AND "plaque OR stenosis." Patients with plaques that could be visualized on 3D time-of-flight (TOF) MRA and axial pregadolinium and postgadolinium contrast T1-weighted spin-echo (T1w-SE) sequences were selected for the study.

Patient exclusion criteria included imaging artifacts secondary to patient motion, metallic susceptibility from medical implants, or complete vessel occlusion. Additionally, intracranial aneurysms, multiple/tandem intracranial stenoses, or presence of moderate to severe atherosclerotic disease in cervical vessels sup- plying the same vascular distribution were also excluded to allow clear association of symptoms with the corresponding intracranial lesion. The retrospective nature of this study prevented patient-specific optimization of imaging parameters such as orientation, section thickness, and coverage. At our institution, the T1w-SE sequences in the MR imaging/MRA brain stroke protocol are performed with the use of axial-oriented imaging and parameters optimized for maximum coverage of the neuroanatomy, limiting vessel coverage to approximately 24 thick (5-mm) axial sections with 1.5-mm gaps.

Although partial volume effects are a concern with 5-mm section imaging, our preliminary studies indicated that cross-sectionally visualized vessels could be successfully evaluated. Because most neuroanatomic MR imaging protocols use axially oriented imaging, vessels parallel to the imaging plane (ie, middle and posterior cerebral arteries), which were more severely affected by partial volume effects, were excluded. Hence, ICAD lesions evaluated in this study were found within the intracranial V4 segment of the vertebral arteries, the basilar artery, supraclinoid ICAs, or the A2 segment of the anterior cerebral artery (ACA). A total of 19 patients age $68.7 \pm 9.6$ years ( 13 men, 6 women) fulfilled the stated criteria and were thus evaluated.

\section{Imaging Evaluation}

Two experienced neuroradiologists (M.C.H. and S.A.A.) performed independent qualitative grading of each ICAD lesion while blinded to the study population: identity, symptoms, DWI abnormalities, and outcomes. Both reviewers confirmed $>70 \%$ stenosis on $3 \mathrm{D}$ TOF and its cross-sectional visualization on precontrast and postcontrast T1w-SE imaging. On the basis of the T1w-SE precontrast and postcontrast imaging, the degree of IPE caused by gadolinium uptake was determined qualitatively and quantitatively by assessment of the degree of enhancement of the plaque/vessel wall. For qualitative assessment, values of 1, 2, 3, 4, or 5 were assigned to each lesion corresponding to "definite nonenhancement," "suspected nonenhancement," "uncertain," "suspected enhancement," and "definite enhancement," respectively. Scores rendered by each independent observer were averaged to determine the final degree of plaque enhancement. A plaque with an average score $>3$ was considered "enhancing" (eg, scores of $5 / 5,5 / 4,4 / 4$, or $4 / 3$ ), and a score $<3$ was considered nonenhancing (eg, scores of $3 / 2,2 / 2,2 / 1$, or $1 / 1$ ). In the event of interobserver disagreement, (eg, a score of $4 / 2$ ), a third independent observer would be used to break the tie.

In a separate and independent evaluation conducted by both observers 1 month after qualitative evaluation, the degree of plaque enhancement was quantified as the relative increase in lesion T1signal postcontrast. Circular ROIs were specifically localized to the lumen of the stenosed vessel and the outer boundary of the vessel wall at the site of the ICAD plaque. The outer wall area was defined and calculated as the difference between the area within the boundary of the vessel wall at the interface with the CSF and the area of the vessel lumen as in Xu et al ${ }^{15}$ and Yuan et $\mathrm{al}^{16}$ and demonstrated in Fig 1 . The percent wall area was defined as the ratio of the outer wall area to the total wall area multiplied by $100 \%$. Percent wall area was used for more meaningful comparison of plaque/vessel wall areas between subgroups containing vessels of different origin and sizes. Quantita- 

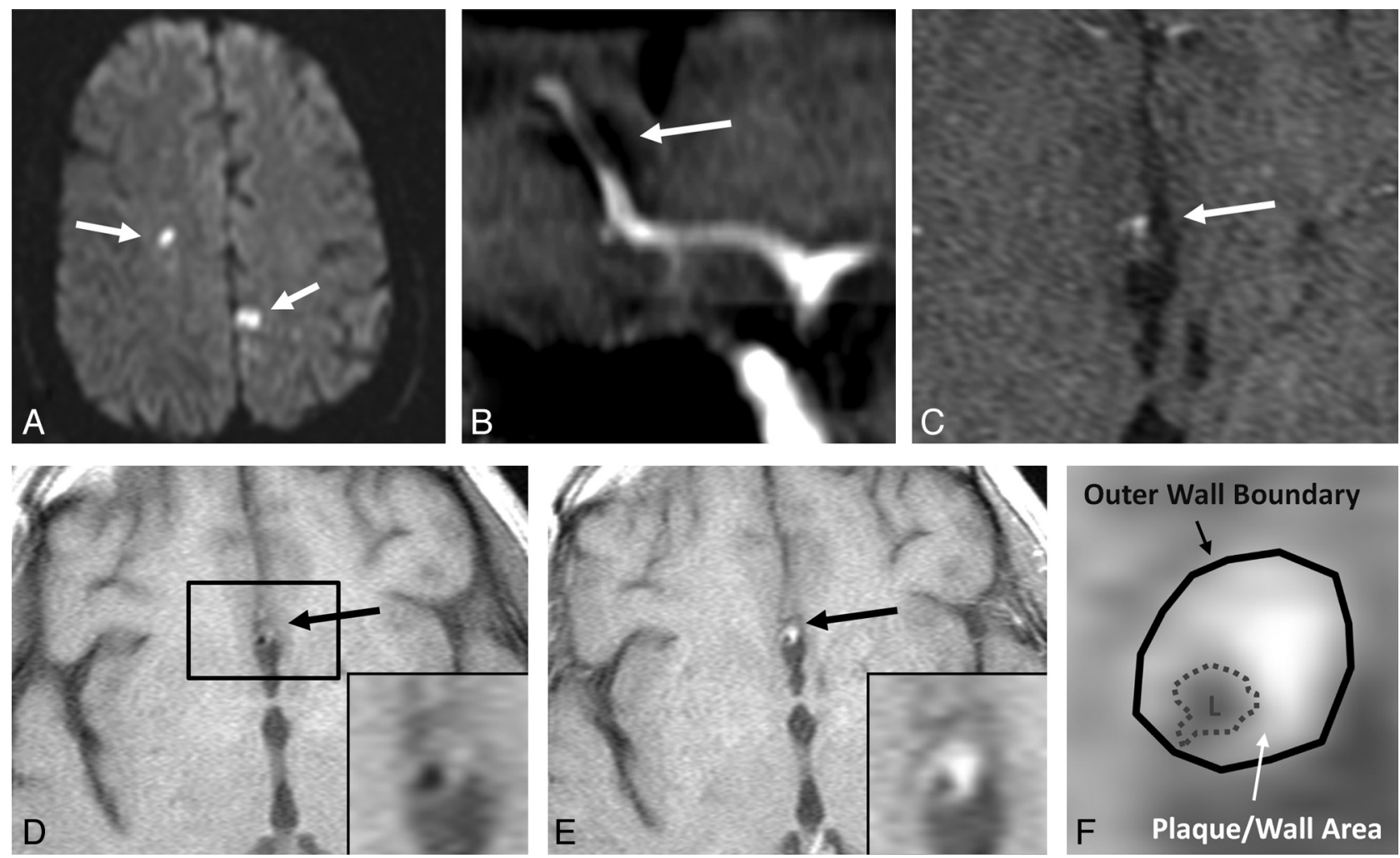

FIG 1. Symptomatic patient with MR DWI (A) demonstrating focal infarcts in the bilateral distributions (white arrows). TOF MR angiography MIP $(B)$ and source images $(C)$ confirm severe stenosis in an A2 azygous ACA segment. Axial T1-weighted-pre $(D)$ and T7-weighted-post contrast (E) images identify enhancing ICAD plaque (inset) involving the azygous A2 ACA segment. Methodology of plaque/wall area measurements are demonstrated $(F)$, with solid black line delineating outer wall boundary and dotted line segmenting the vessel lumen denoted as "L."

tive plaque enhancement was defined as the mean T1 signal intensity in the outer wall area on postcontrast T1w-SE images divided by its precontrast $\mathrm{T} 1$ signal. All T1 signal intensity values in precontrast and postcontrast images were normalized with the signal in the ventricle to control for signal intensity differences between the 2 separate sequences. ROI analysis was conducted with the use of in-house software developed in Matlab (MathWorks, Natick, Massachusetts).

\section{Clinical Evaluation}

All patient neurologic histories, physical examinations, and DWI findings, at the time of diagnostic MR imaging/MRA imaging, were recorded by means of medical record and PACS imaging review. Of the 19 patients, 16 received MR imaging within 24 hours of symptomatic presentations with suspected TIAs or strokes. Routine follow-up stroke protocol MR imaging was performed for an additional 3 patients with history of stroke; all 3 patients harbored severe intracranial stenoses but were asymptomatic at the time of imaging. Presentations with acute ischemic symptoms were classified into anterior or posterior circulation pathology. ${ }^{12}$ A symptomatic ICAD plaque was defined as presenting with acute neurologic (ischemic) symptoms and/or restricted DWI abnormalities (acute watershed, thromboembolic, or perforator infarcts) corresponding to the vascular distribution of the intracranial stenosis. Asymptomatic patients either presented for follow-up imaging because of history of stroke or received first-time imaging in response to symptoms (typically TIAs) that were not associated with the plaque of interest, that is, symptoms that did not correlate to the vascular distribution of the intracranial stenosis. $^{12}$

\section{MR Imaging}

Patients referred to our institution who demonstrated symptoms of TIA or stroke, as determined from neurologic examination, received an MR imaging/MRA stroke imaging protocol. This included the following relevant sequences evaluated in this study: precontrast 3D TOF, DWI, axial T1w-SE before and after a single-dose $(0.1 \mathrm{mmol} / \mathrm{kg})$ injection of a gadolinium-based T1 shortening contrast agent (Magnevist; Bayer HealthCare Pharmaceuticals, Wayne, New Jersey). All patients received postcontrast $\mathrm{T} 1$ imaging within 20 minutes of contrast injection. MR imaging studies were performed on a $1.5 \mathrm{~T}$ scanner (Avanto/Espree; Siemens, Erlangen, Germany) with the use of a receive-only head coil for signal reception. Typical imaging parameters were as follows:

3D TOF. TR/TE $23 \mathrm{~ms} / 5.1 \mathrm{~ms}, 25^{\circ}$ flip angle, $320 \times 284$ matrix, 220-240 mm FOV, voxel size $0.69 \times 0.77 \times 0.63 \mathrm{~mm}$.

Tlw-SE. TR/TE $663 \mathrm{~ms} / 15 \mathrm{~ms}, 90^{\circ}$ flip angle, $256 \times 256$ matrix with 220-240 mm FOV, section thickness $=5 \mathrm{~mm}$, section gap $=$ $1.5 \mathrm{~mm}$, voxel size $0.9 \times 0.9 \times 5 \mathrm{~mm}$.

DWI. TR/TE $4500 / 185 \mathrm{~ms}$, $90^{\circ}$ flip angle, $192 \times 192$ matrix, 220-240 mm FOV, section gap $1.5 \mathrm{~mm}$, voxel size $1.3 \times 1.3 \times 5$ $\mathrm{mm}$.

\section{Statistical Analysis}

The interobserver strength of agreement on qualitative grading of ICAD plaque enhancement was determined by means of quadrat- 
Table 1: Patient demographics, clinical history, and imaging assessment

\begin{tabular}{lcc}
\hline \multicolumn{1}{c}{ Patient Characteristics } & Symptomatic & Asymptomatic \\
\hline$n$ & 10 & 9 \\
Age, y & $66.7(\mathrm{SD}, 11.7 ;$ range, 46-83) & $70.9(\mathrm{SD}, 6.7 ;$ range $60-81)$ \\
Sex & $7 \mathrm{M} ; 3 \mathrm{~F}$ & $6 \mathrm{M} ; 3 \mathrm{~F}$ \\
Active smoker & $1(10 \%)$ & $1(11 \%)$ \\
History of ischemic stroke & $6(60 \%)$ & $0(0 \%)$ \\
TIA & $10(100 \%)$ & $1(11 \%)^{\mathrm{a}}$ \\
Diabetes mellitus & $5(50 \%)$ & $4(44 \%)$ \\
Hypertension & $10(100 \%)$ & $7(78 \%)$ \\
Hyperlipidemia & $9(90 \%)$ & $7(78 \%)$ \\
Statin therapy & $8(80 \%)$ & $5(56 \%)$ \\
Antiplatelet therapy & $6(60 \%)$ & $4(44 \%)$ \\
Hypertension therapy & $8(80 \%)$ & $5(56 \%)$ \\
Atrial fibrillation & $0(0 \%)$ & $0(0 \%)$ \\
Anticoagulant therapy & $2(20 \%)$ & $1(11 \%)$ \\
IPE & $7(70 \%)$ & $1(11 \%)$ \\
\hline
\end{tabular}

${ }^{a}$ History of TIA.

ically weighted Cohen $\kappa$ statistic for categoric variables. Reliabilities $<0.4$ were defined as poor; $0.4-0.75$ as fair to good; and $>0.75$ as excellent. ${ }^{13}$ Interobserver agreement on measurements of plaque/vessel wall areas and quantitative T1 wall enhancement was assessed by means of the Wilcoxon rank sum test for continuous variables. With the use of a paired Student $t$ test, the quantitative measure of $\mathrm{T} 1$ signal enhancement and percentage wall area were compared between symptomatic and asymptomatic lesion cohorts. Logistic regression was used to evaluate IPE as a predictor of ischemic symptoms. A value of $P<.05$ was defined as statistically significant. All calculations were performed with the use of in-house software written in Matlab.

\section{RESULTS}

Initially, 40 patients with severe $(>70 \%)$ intracranial stenoses (confirmed on TOF MRA by both reviewers) were identified for this study. ICAD plaques in 21 patients were excluded for the following reasons: MR imaging studies in 4 patients were nondiagnostic because of severe motion or susceptibility (metallic implants) artifacts, 3 patients had total vessel occlusion, 1 patient had a partially thrombosed fusiform aneurysm distal to the stenosis, and 5 patients had various confounding stenoses in the same vascular distribution. In addition, 8 patients presented with intracranial stenoses involving the MCA or posterior cerebral artery preventing lesion evaluation with the standard 5-mm-thick axial imaging as stated in the Materials and Methods section. All remaining lesions were in the intracranial ICA, A2 ACA, basilar artery, or V4 vertebral arteries, with the stenosis cross-sectionally visualized in the axial plane.

A total of 22 severe ICAD plaques were evaluated independently in 19 patients by 2 neuroradiologists blinded to each subject's clinical data. Table 1 and the On-line Table provide pertinent patient demographics, clinical history, ICAD risk factors, and qualitative and quantitative results for each recruited subject and lesion. In our cohort of $22 \mathrm{ICAD}$ plaques, 10 patients presented with 10 symptomatic plaques and 9 patients harbored 12 asymptomatic plaques. Asymptomatic plaques remained stable, with no attributed ischemic symptoms or acute infarcts on DWI for at least 3 months.

Within our symptomatic cohort, precontrast and postcontrast T1w-SE imaging revealed qualitative contrast enhancement in 7 of $10(70 \%)$ atherosclerotic plaques, correlating with symptomatic presentations and/or acute infarcts on DWI. Conversely, within our asymptomatic cohort, 11 of 12 (92\%) atherosclerotic plaques were determined to be nonenhancing according to precontrast and postcontrast T1w-SE imaging.

Vessels of symptomatic lesions did not have a higher percentage plaque/ vessel wall area compared with asymptomatic lesions at the site of the ICAD plaque $(89.7 \pm 4.0 \%$ versus $86.9 \pm$ $6.1 \%$, respectively, $P=.2$ in Student $t$ test). Furthermore, the cross-sectional areas of plaques in the symptomatic and asymptomatic cohort were not significantly different $\left(12.8 \pm 5.9 \mathrm{~mm}^{2}\right.$ versus $10.8 \pm 4.0 \mathrm{~mm}^{2}$, respectively, $P=.4$, Student $t$ test). The mean increase in quantitative enhancement in the outer wall of symptomatic stenosed vessels at the site of the ICAD plaque was $63 \%$ (precontrast to postcontrast relative signal change of $1.63 \pm 0.28$ ) compared with $23 \%$ in the outer wall of asymptomatic lesions $(1.23 \pm 0.18$ relative signal change). A Student $t$ test found this difference to be significant $(P=.001)$. In addition, a logistic regression model found IPE to be significantly associated with ischemic symptoms or positive DWI findings, that is, IPE-demonstrating plaques were 25 times more likely to be symptomatic $(P=$ .0095).

Interobserver agreement was excellent for determining qualitative plaque enhancement on T1w-SE imaging. A quadratic weighted Cohen $\kappa$ value of 0.82 was found. Quantitative enhancement scores and outer plaque/wall area measurements were also found to be in agreement between both observers because the Wilcoxon signed rank sum test showed insignificant differences between independent measurements $(P=.4$ between separate quantitative enhancement scores and $P=.3$ between separate outer plaque/wall area measurements).

\section{Illustrative Cases}

Patient 2 is a 60-year-old man with history of stroke/TIAs, hyperlipidemia, and hypertension, presenting at the time of imaging with acute lower-extremity weakness and acute infarcts on DWI in the bilateral ACA distributions (Fig $1 A$ ). Hence, the ICAD plaque was determined to be symptomatic. A severe intracranial stenosis involving the azygous A2 branch of the ACA was confirmed on TOF MRA sagittal MIP (Fig 1B) and source images (Fig $1 C)$. Postcontrast enhanced T1w-SE images (Fig $1 E$ ) visualizing the ICAD plaque were graded to be "definitely enhancing" (scored 5), relative to precontrast T1 imaging (Fig $1 D$ ), by both observers. ROIs drawn (Fig $1 F$ ) around the vessel outer wall boundary, segmenting out the lumen, demonstrated quantitative enhancement of $78 \%$ between precontrast and postcontrast images.

Patient 14 is a 75-year-old man with a history of hypertension, hyperlipidemia, and prior vertebrobasilar TIAs/strokes in the posterior circulation but with no active symptoms or acute infarcts on DWI, presenting for follow-up imaging evaluation of 

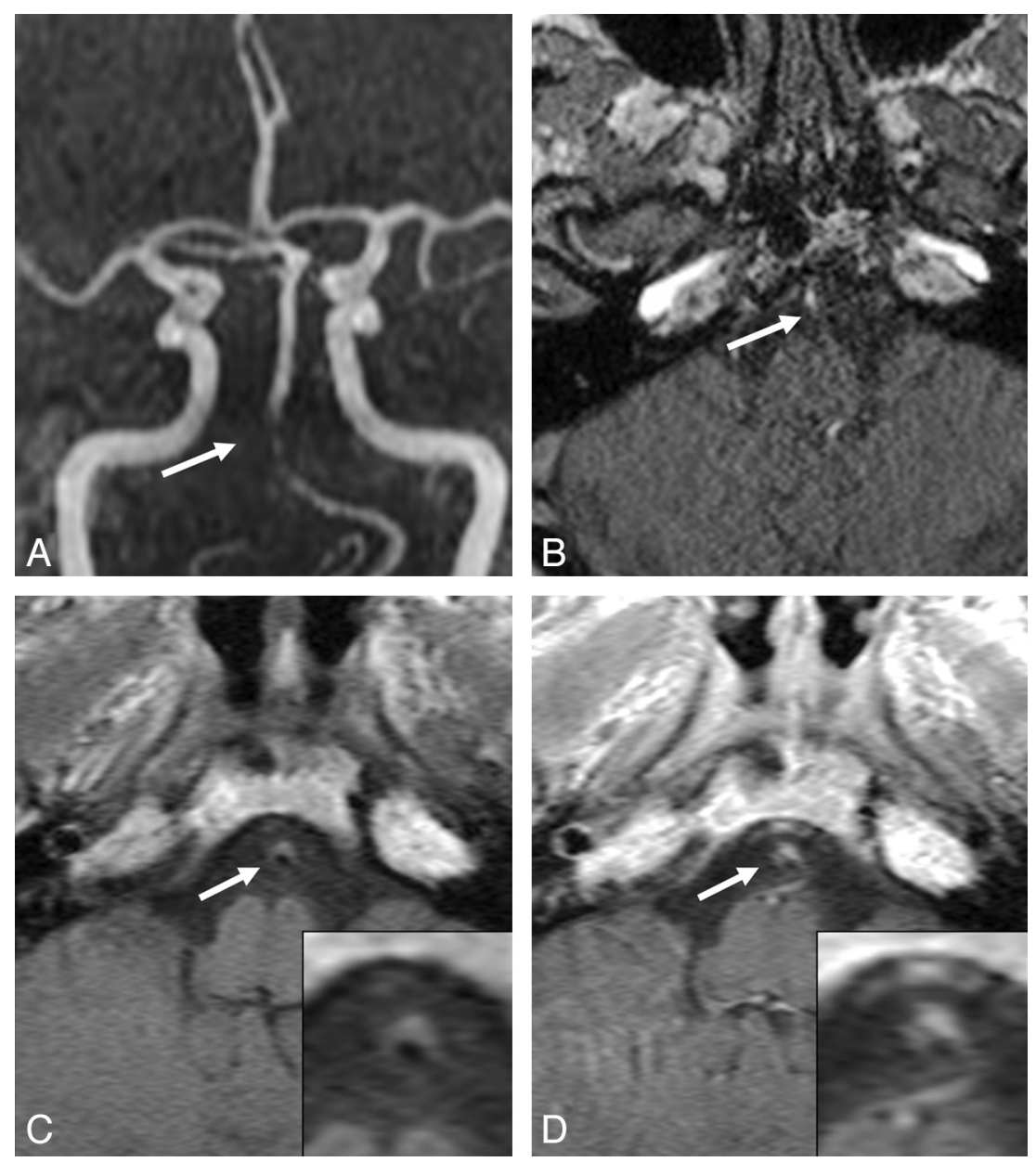

FIG 2. Asymptomatic patient with history of stroke receiving follow-up imaging. TOF MRA reveals severe basilar stenosis in MIP $(A)$ and source images $(B)$. Axial TTWI precontrast $(C)$ and postcontrast $(D)$ demonstrates nonenhancing ICAD plaque.

severe intracranial stenosis of the basilar artery. The patient's ICAD plaque, visualized on TOF MRA MIP (Fig 2A) and source images (Fig $2 B$ ), was subsequently determined to be asymptomatic. Precontrast (Fig 2B) and postcontrast (Fig 2B) T1w-SE imaging demonstrated a nonenhancing atherosclerotic plaque, receiving scores of 2 from both reviewers, with quantitative enhancement of $16 \%$.

\section{DISCUSSION}

In this study of severe ICAD, we found that IPE was strongly associated with symptomatic ischemic presentations. Furthermore, postgadolinium T1w-SE images with the use of conventional, low-resolution MR imaging were capable of reliably detecting IPE (high interobserver agreement; $\kappa=0.82$ ) when the acquisition sections allowed cross-sectional visualization of the vessels of interest. This may indicate that a more robust implementation of this protocol would require sagittal or oblique acquisitions for assessing atherosclerotic disease in the middle cerebral arteries. Given the association between IPE in carotid atherosclerotic disease, plaque destabilization, and thromboembolic events, our study suggests that an analogous process may occur in the intracranial vasculature in the setting of ICAD.
Prior MR imaging and histopathologic studies have shown that vulnerable carotid artery atherosclerotic plaques are characterized by a ruptured fibrous cap, a lipid-rich necrotic core, and IPH. ${ }^{7,17}$ Furthermore, postgadolinium T1 enhancement of symptomatic carotid plaques suggests plaque neovascularization and/or inflammation may predispose plaques to IPH or ischemic/ thromboembolic complications. ${ }^{13,18}$ In fact, Kerwin et $\mathrm{al}^{19}$ introduced several quantitative MR imaging parameters including higher $K^{\text {trans }}$ measurements or adventitial contrast uptake with the use of dynamic contrast-enhanced MR imaging that correlated with macrophage infiltration (inflammation) and neovasculature on subsequent histopathologic analyses. $^{20,21}$

Our study suggests that similar arterial wall pathology in ICAD may be the inciting factor for plaque progression and destabilization. Although neovascularization may be responsible for IPH as the result of rupture of fragile adventitial neovessels resulting in a thrombogenic lipid core, both neovascularization and IPH are associated with the recruitment of inflammatory cells. Conversely, because intracranial vessels usually lack the presence of vasa vasorum, atherosclerotic inflammation may promote a hypoxic environment and stimulate pathologic angiogenic factors such as vascular endo-

thelial growth factor to promote neovascularization, contributing to plaque instability by means of edema, hemorrhage, and rupture. ${ }^{22}$ However, we recommend caution in interpreting our findings in the absence of IPE as stable atherosclerotic lesions. Ischemic complications related to ICAD may also be precipitated through independent mechanisms including thin or ruptured fibrous caps, a large lipidrich necrotic core, and/or IPH.

Previous studies have reported differences in plaque/wall thickening between asymptomatic and symptomatic lesions. ${ }^{15,16}$ This could bias enhancement scores because larger lesions could show greater enhancement. However, we observed no statistically significant differences in total plaque area or percentage wall area within the capacity of low-resolution $1.5 \mathrm{~T}$ MR imaging techniques. We therefore do not believe that greater enhancement in the symptomatic group is attributable to differences in plaque/ wall area size or percentage alone.

A paucity of previous literature has studied ICAD IPE through the use of high-resolution MR imaging techniques but with variable correlations to symptomatic ischemic presentations. ${ }^{9}$ Klein et $\mathrm{al}^{10}$ studied 6 patients with MCA stenoses, but both asymptomatic and symptomatic patients displayed plaque enhancement. Xu et $\mathrm{al}^{11} \mathrm{re}-$ cently characterized a large series of severe MCA stenoses $(>70 \%$ 
occlusion) with high-resolution MR imaging and detected a significantly increased rate of T1 hyperintensity indicative of IPH in symptomatic versus asymptomatic plaques (19.6\% versus $3.2 \%$ ).

The progression and instability of ICAD plaques secondary to neovascularization, IPH, and/or inflammation can present with either symptomatic ischemic or thromboembolic complications. In extracranial carotid atherosclerosis, end-stage hypoperfusion with watershed infarcts to the distal intracranial circulation requires an extensive plaque burden to achieve luminal stenosis. Conversely, acute inflammation/edema or IPH of even moderate ICAD stenoses could achieve significant percentage narrowing in small (diameter $<3 \mathrm{~mm}$ ), fragile intracranial vessels that lack an external elastic lamina and adventitial support, predisposing to watershed ischemia. Alternatively, local IPH and thrombogenic plaque rupture may result in adjacent small perforator occlusions or thromboembolic complications to the distal intracranial vasculature. These mechanisms may be an explanation for the propensity of symptomatic intracranial stenoses to present equivalently with ischemic watershed and thromboembolic infarcts.

Because inflammation is an integral component of atherosclerosis, it may be a transient or undulating process. Identification of active inflammation and/or neovascularization (IPE) could serve as future diagnostic imaging markers to characterize the susceptibility of an ICAD plaque for symptomatic presentations. Furthermore, there may be ramifications for treatment planning in ICAD whereby angioplasty/stent placement could be advocated, delayed, or contraindicated in favor of antiplatelet, anticholesterolemic (statins), novel anti-inflammatory, or anti-angiogenic medical therapies. In fact, statins have been shown to reduce at intervals the quantitative $K^{\text {trans }}$ measurements of adventitial contrast enhancement after 1 year of therapy in cervical carotid atherosclerotic plaques. ${ }^{23}$

Our data suggest that identification of ICAD plaque neovascularization and/or inflammation with postcontrast T1WI may be used to assess lesion vulnerability for symptomatic ischemic or thromboembolic presentations. Interestingly, the failure of the SAMMPRIS trial has been attributed to high periprocedural risk ( $14.7 \%$ mean adverse events in $<30$ days $)$. $^{2}$ The trial was designed to initiate intracranial angioplasty/stent placement early after enrollment of patients presenting with TIAs/strokes ( $<30$ days) to maximize the potential clinical benefit of intervention, but this strategy may be flawed. Early imprudent enrollment with angioplasty/stent placement in the acute setting could disrupt potentially active, inflammatory, and/or thrombogenic atherosclerotic plaques, possibly accounting for the high periprocedural complications (thromboembolism/stroke dissection and vessel wall rupture/intracranial hemorrhage) in the trial.

We propose that future high-resolution and functional MR imaging will assist in better interpreting the vulnerability of ICAD plaques and hence risk stratification of severe intracranial stenoses for improved treatment planning. Future diagnostic imaging studies in ICAD may include high-resolution intracranial plaque visualization with symptomatic correlation, quantitative plaque T1/T2 intensity and contrast enhancement, contrast permeability $\left(K^{\text {trans }}\right)$ measurements, quantitative perfusion MR imaging, and oxygen extraction fraction (PET/MR imaging) parameters to document stage 2 hemodynamic failure and impaired cerebrovascular reserve.
Wall enhancement can be related to vasculitis or inflammation associated with atherosclerotic disease, and we cannot definitively exclude a vasculitic process in our patient cohort. However, because of the advanced age and findings of multiple atherosclerotic risk factors in the studied group as well as no demonstration of clinical or imaging evidence of cerebral vasculitis, the likelihood of a superimposed vasculitic etiology is small.

Our study posed several inherent limitations and challenges. The small sample size limits the power of the statistical results, and, as such, they should be interpreted with caution. Although T1w-SE imaging is an inherently dark-blood technique, slowflow regions within small intracranial vessels adjacent to an atherosclerotic lesion may produce hyperintense signal that could be mistaken for wall enhancement, resulting in false-positive values and reducing the technique's specificity. Delineating boundaries of the vessel lumen by use of an appropriate diffusion-prepared dark-blood imaging technique ${ }^{24,25}$ may mitigate this issue. Ultimately, high-resolution dark-blood imaging sequences, at highfield strengths with improved SNR, should be used to direct imaging to specific vessel morphology and pathology. $8,10,24$

Because of the retrospective nature of this study, we were limited to standard neurovascular anatomic protocols on a $1.5 \mathrm{~T}$ scanner. The use of thick sections $(5 \mathrm{~mm})$ in the T1w-SE acquisitions improved the SNR limitations of the lower field magnet but could cause errors in plaque enhancement evaluation as a result of partial volume averaging. In addition, this protocol did not allow successful evaluation of vessels that projected parallel to the imaging plane, such as the MCAs, producing a selection bias of intracranial plaques. Therefore, we do not advocate conventional MR imaging protocols as sufficient or robust diagnostic tools for intracranial vessel and plaque interrogation.

\section{CONCLUSIONS}

Standard low-resolution neuroanatomic MR imaging may provide useful information for the evaluation of ICAD by demonstrating IPE on precontrast and postcontrast T1w-SE imaging in severely stenotic vessels visualized cross-sectionally in the imaging plane. In these vessels, we found an association between IPE and ischemic/thromboembolic events through the use of conventional MR imaging. However, assessment of the true sensitivity, specificity, and predictive values of IPE in the setting of severe ICAD will require a large, prospective, longitudinal study with the use of high-resolution plaque MR imaging techniques and pathology correlation.

Disclosures: Parmede Vakil—RELATED: Grant: NIH*; UNRELATED: Grants/Grants Pending: NIH. * Richard Bernstein—UNRELATED: Consultancy: Boehringer Ingelheim, Janssen, Medtronic, Medscap, Comments: None relevant to this work; Payment for Lectures (including service on Speakers Bureaus): Boehringer Ingelheim, Janssen, Medtronic, Comments: None relevant; Payment for Development of Educational Presentations: Janssen Pharmaceuticals, Medscape, Boehringer Ingelheim. Timothy Carroll—RELATED: Grant: $\mathrm{NIH}$, Comments: Two separate NIH grants supported this work; UNRELATED: Grants/ Grants Pending: NIH (*money paid to institutions).

\section{REFERENCES}

1. Chimowitz MI, Lynn MJ, Howlett-Smith H, et al. Comparison of warfarin and aspirin for symptomatic intracranial arterial stenosis. N Engl J Med 2005;352:1305-16

2. Chimowitz MI, Lynn MJ, Derdeyn CP, et al. Stenting versus aggressive medical therapy for intracranial arterial stenosis. $N$ Engl J Med 2011;365:993-1003 
3. Yuan C, Mitsumori LM, Beach KW, et al. Carotid atherosclerotic plaque: noninvasive MR characterization and identification of vulnerable lesions. Radiology 2001;221:285-99

4. Wasserman BA, Wityk RJ, Trout HH 3rd, et al. Low-grade carotid stenosis: looking beyond the lumen with MRI. Stroke 2005;36:2504-13

5. Kerwin WS, O’Brien KD, Ferguson MS, et al. Inflammation in carotid atherosclerotic plaque: a dynamic contrast-enhanced MR imaging study. Radiology 2006;241:459-68

6. Saam T, Hatsukami TS, Takaya N, et al. The vulnerable, or high-risk, atherosclerotic plaque: noninvasive MR imaging for characterization and assessment. Radiology 2007;244:64-77

7. Spagnoli LG, Mauriello A, Sangiorgi G, et al. Extracranial thrombotically active carotid plaque as a risk factor for ischemic stroke. JAMA 2004;292:1845-52

8. Vergouwen MD, Silver FL, Mandell DM, et al. Eccentric narrowing and enhancement of symptomatic middle cerebral artery stenoses in patients with recent ischemic stroke. Arch Neurol 2011;68:338-42

9. Swartz RH, Bhuta SS, Farb RI, et al. Intracranial arterial wall imaging using high-resolution 3-Tesla contrast-enhanced MRI. Neurology 2009;72:627-34

10. Klein IF, Lavallee PC, Touboul PJ, et al. In vivo middle cerebral artery plaque imaging by high-resolution MRI. Neurology 2006;67:327-29

11. $\mathrm{Xu} \mathrm{WH}, \mathrm{Li} \mathrm{ML}$, Gao $\mathrm{S}$, et al. Middle cerebral artery intraplaque hemorrhage: prevalence and clinical relevance. Ann Neurol 2012;71: 195-98

12. Greenberg MS. Handbook of Neurosurgery. New York: Thieme Medical Publishers; 2010

13. Qiao Y, Steinman DA, Qin Q, et al. Intracranial arterial wall imaging using three-dimensional high isotropic resolution black blood MRI at 3.0 Tesla. J Magn Reson Imaging 2011;34:22-30

14. Skarpathiotakis M, Mandell DM, Swartz RH, et al. Intracranial atherosclerotic plaque enhancement in patients with ischemic stroke. AJNR Am J Neuroradiol 2013;34:299-304

15. $\mathrm{Xu} \mathrm{WH}, \mathrm{Li} \mathrm{ML}, \mathrm{Gao}$, et al. In vivo high-resolution MR imaging of symptomatic and asymptomatic middle cerebral artery atherosclerotic stenosis. Atherosclerosis 2010;212:507-11

16. Yuan C, Beach KW, Smith LH Jr, et al. Measurement of atherosclerotic carotid plaque size in vivo using high resolution magnetic resonance imaging. Circulation 1998;98:2666-71

17. Redgrave JN, Lovett JK, Gallagher PJ, et al. Histological assessment of 526 symptomatic carotid plaques in relation to the nature and timing of ischemic symptoms: the Oxford plaque study. Circulation 2006;113:2320-28

18. McCarthy MJ, Loftus IM, Thompson MM, et al. Angiogenesis and the atherosclerotic carotid plaque: an association between symptomatology and plaque morphology. J Vasc Surg 1999;30:261-68

19. Kerwin WS, Oikawa M, Yuan C, et al. MR imaging of adventitial vasa vasorum in carotid atherosclerosis. Magn Reson Med 2008;59:507-14

20. Kerwin W, Hooker A, Spilker M, et al. Quantitative magnetic resonance imaging analysis of neovasculature volume in carotid atherosclerotic plaque. Circulation 2003;107:851-56

21. Cai J, Hatsukami TS, Ferguson MS, et al. In vivo quantitative measurement of intact fibrous cap and lipid-rich necrotic core size in atherosclerotic carotid plaque: comparison of high-resolution, contrast-enhanced magnetic resonance imaging and histology. Circulation 2005;112:3437-44

22. Moreno PR, Purushothaman KR, Sirol M, et al. Neovascularization in human atherosclerosis. Circulation 2006;113:2245-52

23. Dong L, Kerwin WS, Chen H, et al. Carotid artery atherosclerosis: effect of intensive lipid therapy on the vasa vasorum-evaluation by using dynamic contrast-enhanced MR imaging. Radiology 2011;260:224-31

24. Ryu CW, Jahng GH, Kim EJ, et al. High resolution wall and lumen MRI of the middle cerebral arteries at 3 Tesla. Cerebrovasc Dis 2009;27:433-42

25. Koktzoglou I, Kirpalani A, Carroll TJ, et al. Dark-blood MRI of the thoracic aorta with 3D diffusion-prepared steady-state free precession: initial clinical evaluation. AJR Am J Roentgenol 2007; 189:966-72 